\title{
Model of Combined IPT and NNLVQ for Classification of Healthy and Sick Broilers In Terms of Avian Influenza
}

\author{
Ahmet Kayabasi ${ }^{1 *}$ (D) \\ ${ }^{1 *}$ Karamanoglu Mehmetbey University, Electrical and Electronics Engineering Department, 70100, Karaman, Turkey. (e-mail: ahmetkayabasi@kmu.edu.tr).
}

\section{ARTICLE INFO}

Received: Feb., 22. 202

Revised: Jul., 26. 2021

Accepted: Aug., 03. 2021

\section{Keywords:}

Broiler chicken

Classification

Avian influenza

Neural network

Learning vector quantization

Corresponding author: Ahmet Kayabasi

ISSN: 2536-5010 | e-ISSN: 2536-5134

DOI: https://doi.org/10.36222/ejt.884730

\section{ABSTRACT}

Poultry meat is an important and economical protein source in providing the animal protein requirement for human nutrition. The poultry diseases such as avian influenza that are a feature of fast-spread in farms seriously threatens both the economy and human health. Avian influenza must be detected early because it spreads rapidly. Earlier detection of poultry diseases has become more possible with the development of systems combining image processing techniques (IPTs) and artificial intelligence techniques (AITs). In this study, the neural network (NN) based model using learning vector quantization (LVQ) structure is proposed for the classification of broiler chickens as healthy and sick. In the literature, seven main visual feature parameters that indicate the health status of broilers were acquired through the IPTs. The data set includes seven visual features is used for training, testing and validating process of the NNLVQ model. The classification performance of the neural network (NN) using learning vector quantization (NNLVQ) is compared with IPT concerning its efficiency and accuracy. In the training process, the NNLVQ model classifies the broilers in terms of avian influenza with an accuracy error (AE) of $0.384 \%$. The results point out that, the IPT based application using NNLVQ is successfully classified the broilers in terms of their health conditions.

\section{INTRODUCTION}

The poultry meat is seen as an important and economical protein source in providing the animal protein needs required for human nutrition with its low fat and high protein content. Poultry meat consumption has been increased in recent years due to cheaper and shorter production times than red meat [1]. Therefore, poultry meat production increased to second place after pork in meat production. To meet this need, broiler chickens are grown which are fast-growing, better utilizing than feed and producing high-quality carcass. The most important factor in poultry farming is the health and regular control of poultry. The prevalence of poultry diseases has seriously affected poultry farming in recent years. This situation poses a threat not only for economic reasons but also for human health. Today, the diagnosis of poultry diseases is performed manually with the observation of the veterinarian and using various laboratory tests. However, manual processes are time-consuming, difficult, and yet fail to detect some of the diseases. Therefore, rapid detection of poultry diseases has become an important issue in broiler breeding. The automatic detection of broiler diseases with the help of image processing techniques (IPTs) has been an important topic in the point of fast diagnosis. For this issue, the neural network (NN) using learning vector quantization (LVQ) can be combined with IPTs.

The several studies regarding the diagnosis of broiler diseases using NNs combined IPT have been proposed in the literature [2]-[8]. In [2], it was comparatively determined by different machine learning algorithms that the broiler chickens were healthy or sick for avian influenza disease and the most successful result was obtained with a support vector machine (SVM). The data set used in machine learning algorithms was created by computer vision. The body weights of live broilers were estimated by using IPT analysis by Mollah et al. [3]. The estimated weights and manual measurement results were shown to be very close to each other. In [4], different artificial intelligence techniques (AITs) were used to estimate intestinal broiler microflora. The results show that the Enterobacteriaceae population was predicted better than the lactic acid bacteria with the proposed models. A novel algorithm of image analysis was investigated for early detection of lameness for broilers by Aydin and some feature variables of broilers were detected by the proposed algorithm [5]. In [6], the welfare status in commercial broiler breeders was assessed by the data mining algorithms combined with IPT and results were obtained successfully. The NNs such as artificial neural network (ANN) and adaptive neuro-fuzzy 
inference system (ANFIS) were proposed to predict chick body mass and more successful results were achieved with ANN by Ferraz et al. [7]. In [8], the weight prediction of broiler chickens five regression model integrated with $3 \mathrm{D}$ computer vision was used and the best result was obtained with the Bayesian ANN model.

In this study, the NN using LVQ (NNLVQ) is modeled to classify broiler chickens as healthy and sick in terms of avian influenza infection. In the literature, the data set includes 7 main visual feature parameters that indicate the health status of broilers were acquired through the IPTs [9]. These visual parameters are concavity, skeleton attitude angle, skeleton splicing angle and shape features (area-linear rate, elongation and circularity). About seven visual features, the 300 data sets were created, 150 of which were healthy and 150 of sick broilers [9]. The accuracy of the models is determined by selected 260 training, 20 testing and 20 validating broilers data set and their performances of classification are compared to each other. In the training process, the NNLVQ model successfully classifies the broilers as healthy and sick with an accuracy of $99.616 \%$.

\section{DATA SET}

The automatic classification application based on NNs is carried out through a data set reported elsewhere [9]. containing 7 main visual feature parameters of broiler chickens. In the literature, four to six weeks old broiler chickens were divided into two groups and placed in isolator cages [2]-[9]. Ten of the twenty R381 group broilers were vaccinated with $0.1 \mathrm{~mL}$ volume of 106 EID50 H5N2 avian influenza virus (R381 / 2008) and the other ten were intranasally injected with $0.1 \mathrm{~mL}$ phosphate-buffered saline (PBS). Clinical symptoms of avian influenza were observed in twenty broilers after 14 days [2]-[9]. As shown in Figure 1, the images of broilers were captured with a resolution of 640 by 480 pixels by using a Logitech C922 CCD camera and image processing was performed using an algorithm based on VS2013 and OpenCV 2.4.13 [2]. To calculate the skeletal structure of the broiler, the algorithm only extracts the image of the broiler from the complex background as shown in Figure 2. The eigenvectors are determined according to the features such as concavity, skeleton attitude angle, skeleton splicing angle and shape features [2]-[9]. Briefly, the process for obtaining data on the visual properties of the broiler is shown as topology in Figure 3 .

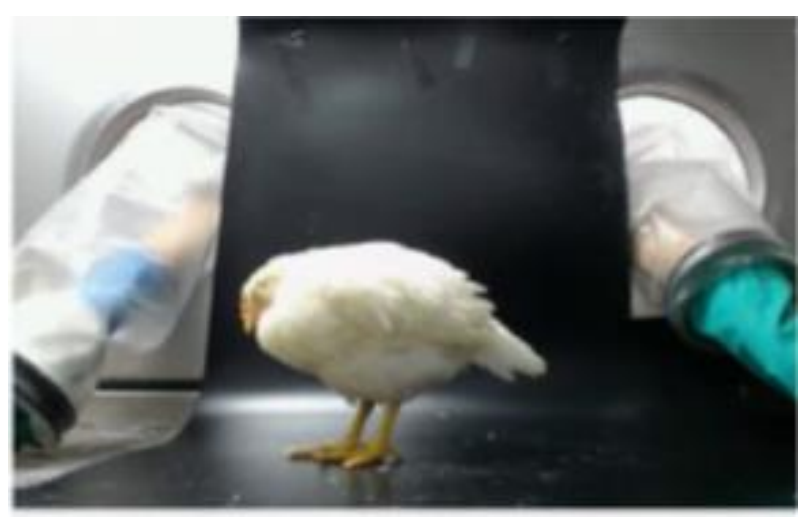

Figure 1. Image of broilers capture environment [2]

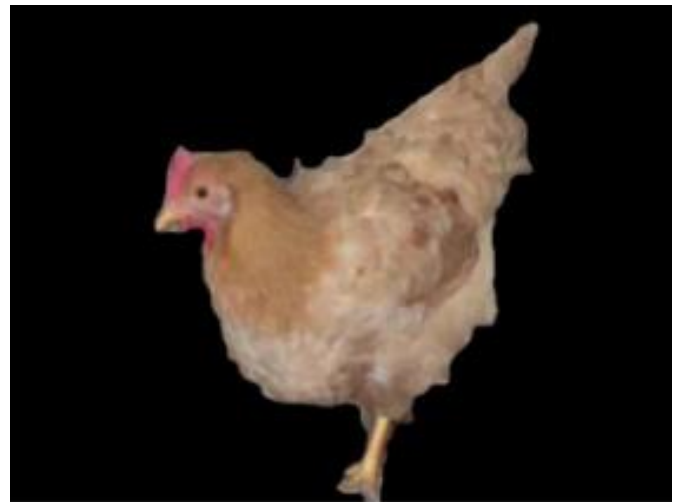

Figure 2. Image of extracted broiler from the background [2]

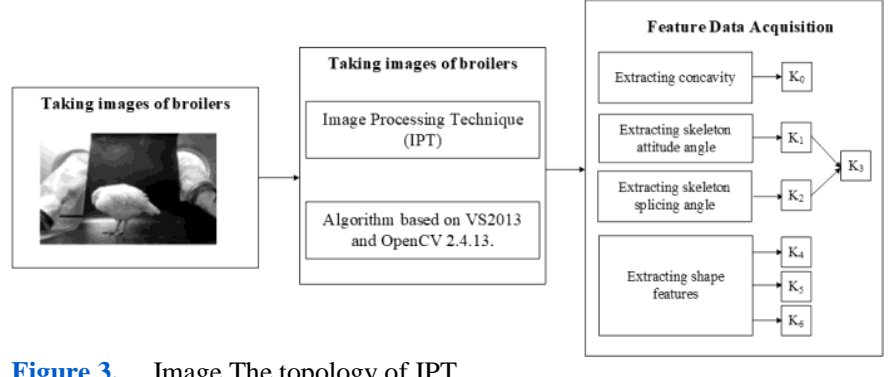

Figure 3. Image The topology of IPT

\subsection{Broiler feature extraction}

In this section, the extraction of the features of broilers will be briefly summarized according to what is described in Zhuang et al. [2]. Concavity, skeleton attitude angle, skeleton splicing angle, area-linear ratio, elongation and circularity were named $K_{0}, K_{1}, K_{2}, K_{4}, K_{5}$ and $K_{6}$ respectively [2]. $K_{3}$ was obtained by using the methods of skeleton simplification and skeleton splicing [2].

$$
\begin{aligned}
& K_{0}=\stackrel{\circ}{\mathrm{a}}_{i=1}^{n} \frac{b\left(s_{1}, s_{2}\right) l\left(s_{1}\right) l\left(s_{2}\right)}{\left[l\left(s_{1}\right)+l\left(s_{2}\right)\right] r}
\end{aligned}
$$

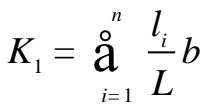

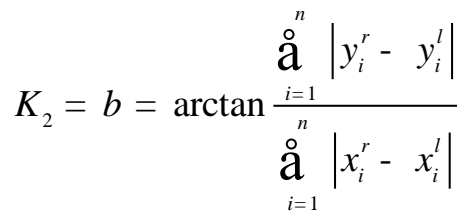

$$
\begin{aligned}
& K_{4}=\frac{S}{C} \\
& K_{5}=\frac{H}{W} \\
& K_{6}=\frac{4 p S}{C^{2}}
\end{aligned}
$$

In the Figure 4, 2D scattering of the broilers features is demonstrated to show how healthy and sick broilers 
discriminate among each other by the feature parameters. In addition, the graphs of all feature parameters $\left(K_{0}, K_{1}, K_{2}, K_{4}, K_{5}\right.$ and $K_{6}$ ) are plotted in Figure 5. It is observed that healthy and sick broilers distinctly cluster for the visual feature parameters.

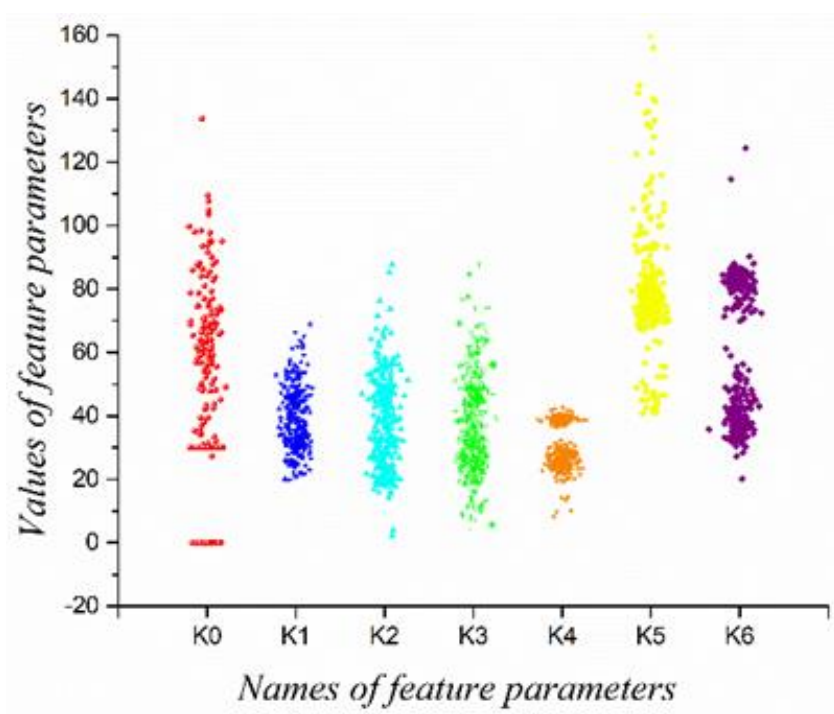

Figure 4. 2D scattering of 300 broilers according to feature parameters

\section{NEURAL NETWORK}

The NN currently provides the best solutions to many problems in image recognition. The $\mathrm{NN}$ interprets the raw input by labeling or clustering with a kind of machine perception. It helps to group unlabeled data according to similarities between sample inputs and classify data when they have a data set labeled for training [2], [3], [13], [4]-[8], [10]-[12]. The modeling and training of the NNLVQ for the classification of broilers in terms of avian influenza are described below.

\subsection{Modelling and training of neural network}

The NNLVQ is modeled and trained with appropriate set parameters according to the topology illustrated in Figure 6. The computer used in this study has property of Intel Core i7 CPU with $3.1 \mathrm{GHz}$ and $8 \mathrm{~GB}$ DDR3 RAM. According to the result of the training, the model is updated and the training is repeated. Modeling and training processes are carried out one after the other to try to obtain the lowest classification accuracy error. The NNLVQ model numerically calculates the outputs according to accuracy error (AE) in given Eq. (7).

The NN consists of neurons that are organized into different layers. These neurons containing a non-linear type of functions are mutually connected by synaptic weights. These weights increase or decrease to output closer to target throughout the training process [11]-[13]. As shown in Figure 7, along with the set parameters given in Table 1, NNLVQ is designed to classify the broilers into "healthy" or "sick" according to the feature parameters. The NNLVQ model is constructed with an input layer having 7 neurons, one hidden layer having 5 neurons and one output layer have 2 neurons.

$$
A E=\frac{\text { Error } x 100}{\text { Actual value }}
$$
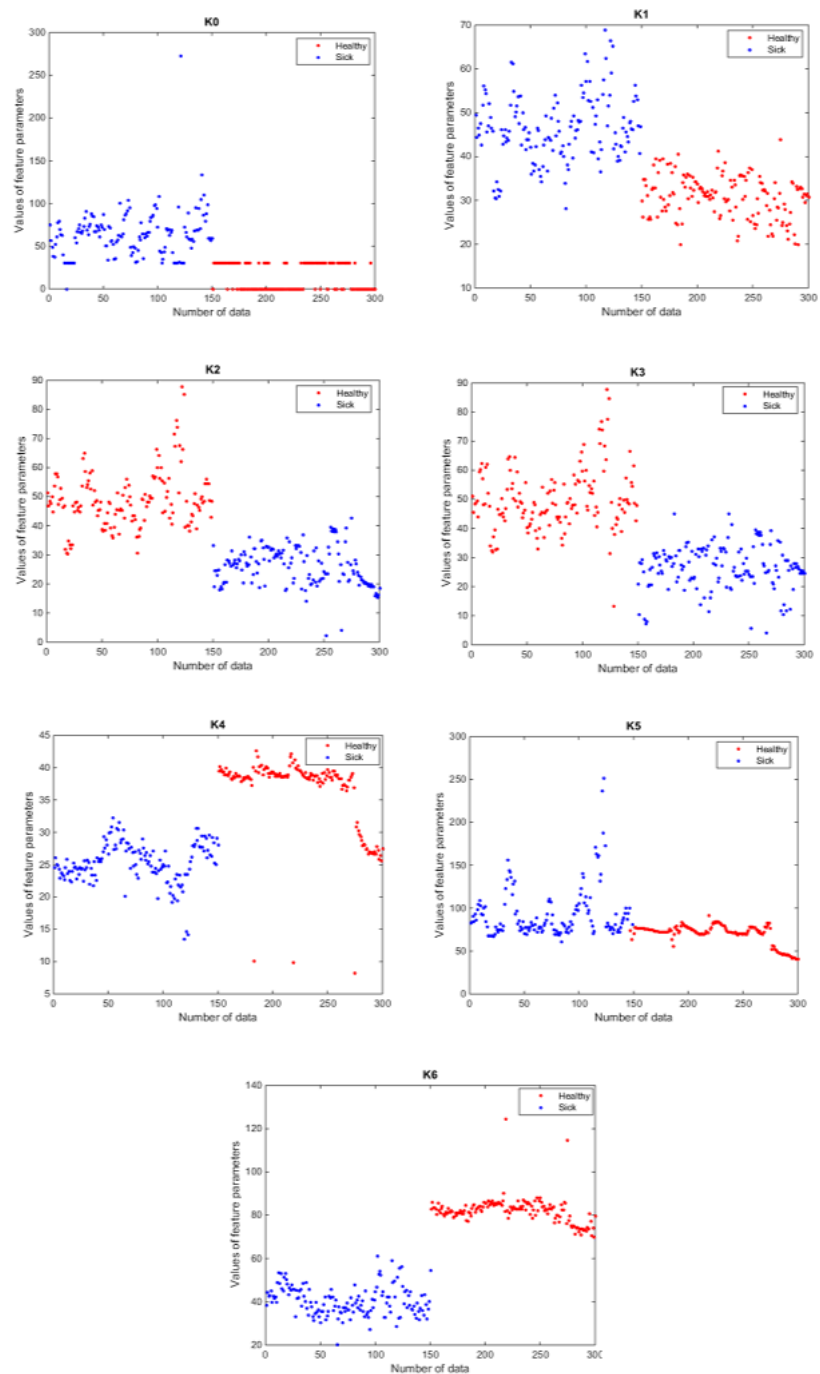

Figure 5. Separately graphs of feature parameters

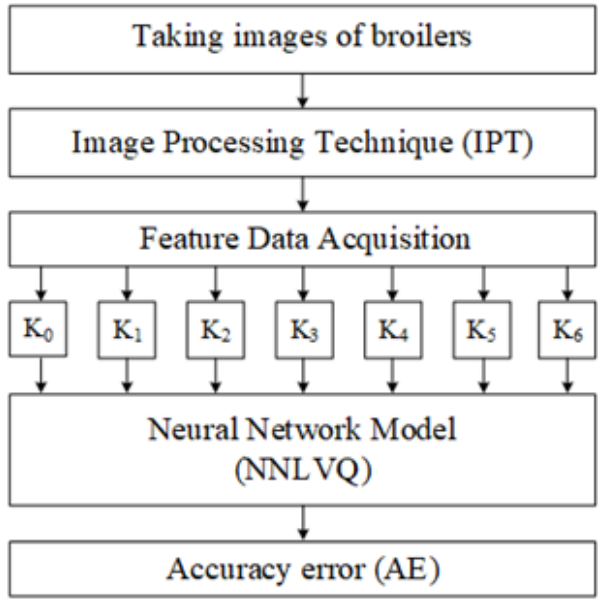

Figure 6. Topology of the NNLVQ model

TABLE I

THE SET PARAMETERS OF THE NNLVQ MOdEL

\begin{tabular}{clc}
\hline Model & \multicolumn{1}{c}{ Parameters } & Set type/value \\
\hline & Epochs & 200 \\
& Minimum gradient descent & $10^{-7}$ \\
$\mathrm{ANN}_{\mathrm{LVQ}}$ & Learning rate & 0.02 \\
& Validation checks & 7 \\
& Output class percentage & $0.5,05$ \\
\hline
\end{tabular}




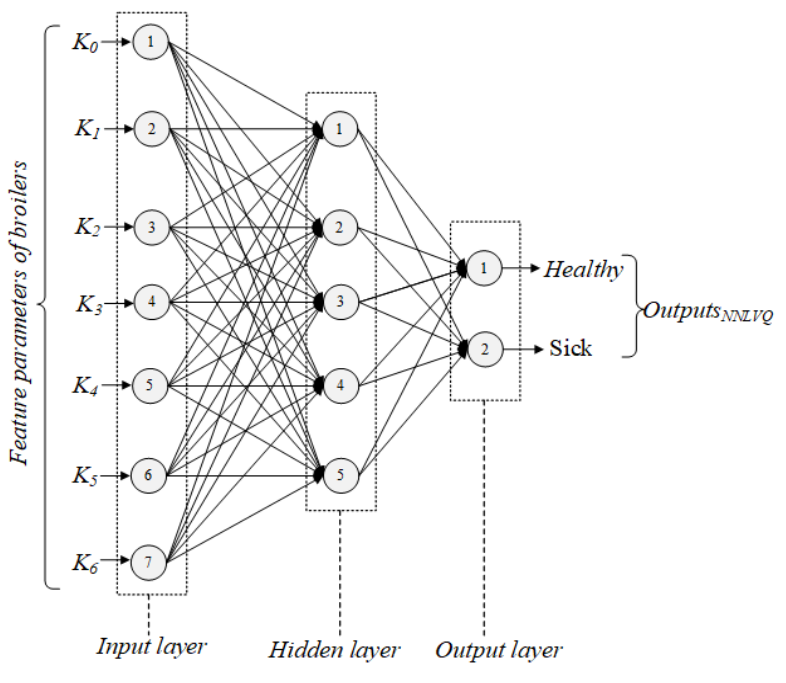

Figure 7. Structure of the NNLVQ model

The 260 visual data of broilers randomly selected from 300 data are used for the training of NNLVQ. The only one of 260 data sets used for the training process are incorrectly classified in the NNLVQ model.

\subsection{Testing and validating of neural network}

The data of 20 broilers' visual features is used to test the accuracy of the NNLVQ. The tabulated in Table 2, 20 test data are randomly selected among 300 broiler visual features and are not utilized during the training phase. The results of NNLVQ model are tabulated in Table 3 for testing process. Only one of the 20 data sets used for the testing process is misclassified in the NNLVQ model.

TABLE II

The Used Dataset In The Testing Process

\begin{tabular}{cccccccc}
\hline Sample & \multicolumn{6}{c}{ Broiler visual feature parameters } \\
\cline { 2 - 8 }$\#$ & $K_{0}$ & $K_{1}$ & $K_{2}$ & $K_{3}$ & $K_{4}$ & $K_{5}$ & $K_{6}$ \\
\hline 1 & 63.107 & 45.511 & 47.432 & 48.280 & 24.506 & 92.983 & 49.032 \\
2 & 77.059 & 56.150 & 57.923 & 60.245 & 23.494 & 104.790 & 41.570 \\
3 & 94.870 & 52.251 & 53.972 & 53.208 & 27.032 & 106.771 & 39.533 \\
4 & 48.201 & 40.805 & 44.882 & 52.308 & 21.656 & 102.395 & 44.597 \\
5 & 88.663 & 38.896 & 40.130 & 13.331 & 27.826 & 74.370 & 45.486 \\
6 & 30.000 & 30.537 & 30.441 & 32.421 & 25.180 & 67.822 & 53.184 \\
7 & 42.109 & 40.774 & 41.463 & 43.884 & 22.894 & 74.899 & 31.859 \\
8 & 57.653 & 43.820 & 45.626 & 45.525 & 28.944 & 76.513 & 38.068 \\
9 & 56.507 & 44.460 & 46.838 & 45.537 & 26.090 & 83.886 & 44.182 \\
10 & 71.938 & 46.576 & 48.617 & 48.596 & 26.984 & 84.884 & 34.354 \\
11 & 0.000 & 33.062 & 27.811 & 27.422 & 40.251 & 79.293 & 84.501 \\
12 & 0.000 & 25.632 & 27.911 & 27.911 & 39.089 & 68.627 & 86.474 \\
13 & 30.000 & 30.170 & 31.432 & 31.776 & 37.738 & 71.717 & 80.250 \\
14 & 0.000 & 38.272 & 28.407 & 32.292 & 38.397 & 72.330 & 80.937 \\
15 & 30.000 & 30.656 & 31.446 & 31.735 & 38.659 & 74.510 & 81.526 \\
16 & 0.000 & 37.426 & 25.710 & 27.892 & 40.695 & 71.698 & 81.854 \\
17 & 0.000 & 27.892 & 26.842 & 11.737 & 29.655 & 52.381 & 74.457 \\
18 & 0.000 & 35.241 & 28.457 & 28.457 & 39.116 & 80.000 & 82.462 \\
19 & 0.000 & 30.149 & 32.986 & 32.986 & 38.708 & 83.505 & 82.744 \\
20 & 0.000 & 37.808 & 27.126 & 29.947 & 38.190 & 72.195 & 80.570 \\
\hline
\end{tabular}

The proposed NNLVQ model is validated with the remaining 20 broiler data given in Table 4 . All 20 data sets used for the validating process are correctly classified in the NNLVQ model. As it is seen from the validating results given in the Table 5, the proposed model can be successfully implemented to such classification of broilers as healthy or sick.
TABLE III

THE Results Of NNLVQ MODEL IN THE TEST PROCESS

\begin{tabular}{cccccc}
\hline \multirow{2}{*}{$\#$} & \multicolumn{2}{c}{ Target } & \multicolumn{2}{c}{ Numerical outputs } & Classification \\
\cline { 3 - 6 } & \multicolumn{2}{c}{1.000} & 0.000 & 1.000 & NNLVQ \\
\hline 1 & 0.000 & 1.000 & 0.000 & 1.000 & Healthy \\
2 & 0.000 & 1.000 & 0.000 & 1.000 & Healthy \\
3 & 0.000 & 1.000 & 1.000 & Healthy \\
4 & 0.000 & 1.000 & 0.000 & 1.000 & Healthy \\
5 & 0.000 & 1.000 & 0.000 & 1.000 & Sick \\
6 & 0.000 & 1.000 & 1.000 & 0.000 & Healthy \\
7 & 0.000 & 1.000 & 0.000 & 1.000 & Healthy \\
8 & 0.000 & 1.000 & 0.000 & 1.000 & Healthy \\
9 & 0.000 & 1.000 & 0.000 & 1.000 & Healthy \\
10 & 0.000 & 1.000 & 0.000 & 1.000 & Sick \\
11 & 1.000 & 0.000 & 1.000 & 0.000 & Sick \\
12 & 1.000 & 0.000 & 1.000 & 0.000 & Sick \\
13 & 1.000 & 0.000 & 1.000 & 0.000 & Sick \\
14 & 1.000 & 0.000 & 1.000 & 0.000 & Sick \\
15 & 1.000 & 0.000 & 1.000 & 0.000 & Sick \\
16 & 1.000 & 0.000 & 1.000 & 0.000 & Sick \\
17 & 1.000 & 0.000 & 1.000 & 0.000 & Sick \\
18 & 1.000 & 0.000 & 1.000 & 0.000 & Sick \\
19 & 1.000 & 0.000 & 1.000 & 0.000 & Sick \\
20 & 1.000 & 0.000 & 1.000 & 0.000 & \\
\hline
\end{tabular}

TABLE IV

The Used Dataset In The Validating Process

\begin{tabular}{cccccccc}
\hline Sample & \multicolumn{7}{c}{ Broiler visual feature parameters } \\
\cline { 2 - 8 }$\#$ & $K_{0}$ & $K_{1}$ & $K_{2}$ & $K_{3}$ & $K_{4}$ & $K_{5}$ & $K_{6}$ \\
\hline 1 & 73.249 & 42.214 & 44.400 & 45.639 & 27.376 & 77.211 & 31.599 \\
2 & 35.776 & 47.980 & 49.050 & 49.021 & 25.439 & 79.237 & 38.477 \\
3 & 32.124 & 33.947 & 36.189 & 36.621 & 28.928 & 71.260 & 47.783 \\
4 & 69.812 & 53.009 & 56.221 & 56.280 & 19.768 & 112.752 & 39.254 \\
5 & 88.663 & 38.896 & 40.130 & 13.331 & 27.826 & 74.370 & 45.486 \\
6 & 50.140 & 36.713 & 38.415 & 41.469 & 30.807 & 77.000 & 38.730 \\
7 & 63.107 & 45.511 & 47.432 & 48.280 & 24.506 & 92.983 & 49.032 \\
8 & 59.294 & 46.962 & 48.768 & 48.673 & 25.266 & 73.646 & 32.114 \\
9 & 48.201 & 40.805 & 44.882 & 52.308 & 21.656 & 102.395 & 44.597 \\
10 & 30.000 & 65.139 & 85.255 & 84.659 & 24.316 & 172.881 & 56.283 \\
11 & 30.000 & 24.690 & 23.574 & 23.574 & 39.190 & 70.202 & 86.018 \\
12 & 30.000 & 30.656 & 31.446 & 31.735 & 38.659 & 74.510 & 81.526 \\
13 & 0.000 & 30.149 & 32.986 & 32.986 & 38.708 & 83.505 & 82.744 \\
14 & 0.000 & 31.578 & 30.196 & 32.819 & 38.687 & 71.212 & 85.141 \\
15 & 0.000 & 37.808 & 27.126 & 29.947 & 38.190 & 72.195 & 80.570 \\
16 & 0.000 & 34.013 & 38.177 & 38.177 & 38.914 & 77.500 & 80.801 \\
17 & 30.000 & 30.170 & 31.432 & 31.776 & 37.738 & 71.717 & 80.250 \\
18 & 0.000 & 27.487 & 17.788 & 16.077 & 40.307 & 70.531 & 84.474 \\
19 & 0.000 & 35.241 & 28.457 & 28.457 & 39.116 & 80.000 & 82.462 \\
20 & 30.000 & 22.279 & 23.376 & 25.104 & 36.441 & 70.202 & 76.827 \\
\hline
\end{tabular}

TABLE V

The Results Of NNLVQ Model In The VALidAting Process

\begin{tabular}{cccccc}
\hline \multirow{2}{*}{$\#$} & \multicolumn{2}{c}{ Target } & \multicolumn{2}{c}{ Numerical outputs } & Classification \\
\cline { 3 - 5 } & \multicolumn{2}{c}{ NNLVQ } & NNLVQ \\
\hline 1 & 0.000 & 1.000 & 0.000 & 1.000 & Healthy \\
2 & 0.000 & 1.000 & 0.000 & 1.000 & Healthy \\
3 & 0.000 & 1.000 & 0.000 & 1.000 & Healthy \\
4 & 0.000 & 1.000 & 0.000 & 1.000 & Healthy \\
5 & 0.000 & 1.000 & 0.000 & 1.000 & Healthy \\
6 & 0.000 & 1.000 & 1.000 & 0.000 & Sick \\
7 & 0.000 & 1.000 & 0.000 & 1.000 & Healthy \\
8 & 0.000 & 1.000 & 0.000 & 1.000 & Healthy \\
9 & 0.000 & 1.000 & 0.000 & 1.000 & Healthy \\
10 & 0.000 & 1.000 & 0.000 & 1.000 & Healthy \\
11 & 1.000 & 0.000 & 1.000 & 0.000 & Sick \\
12 & 1.000 & 0.000 & 1.000 & 0.000 & Sick \\
13 & 1.000 & 0.000 & 1.000 & 0.000 & Sick \\
14 & 1.000 & 0.000 & 1.000 & 0.000 & Sick \\
15 & 1.000 & 0.000 & 1.000 & 0.000 & Sick \\
16 & 1.000 & 0.000 & 1.000 & 0.000 & Sick \\
17 & 1.000 & 0.000 & 1.000 & 0.000 & Sick \\
18 & 1.000 & 0.000 & 1.000 & 0.000 & Sick \\
19 & 1.000 & 0.000 & 1.000 & 0.000 & Sick \\
20 & 1.000 & 0.000 & 1.000 & 0.000 & Sick \\
\hline
\end{tabular}

It is seen from these results that the proposed NNLVQ model based on IPT is successful. This model can be used to 
automatically detect sick broilers in a farm as shown in Figure 8. In this regard, images taken at certain intervals with cameras on a farm can be analyzed using NNLVQ. After determining the coordinate of the sick broiler, it can be taken to another area by removing the broiler with a 3-dimensional movable mechanism. In this way, early screening can be done by making an instant scan and the spread can be prevented. Also, the presented models can be easily integrated into farm industry to automatically classify of different animal.
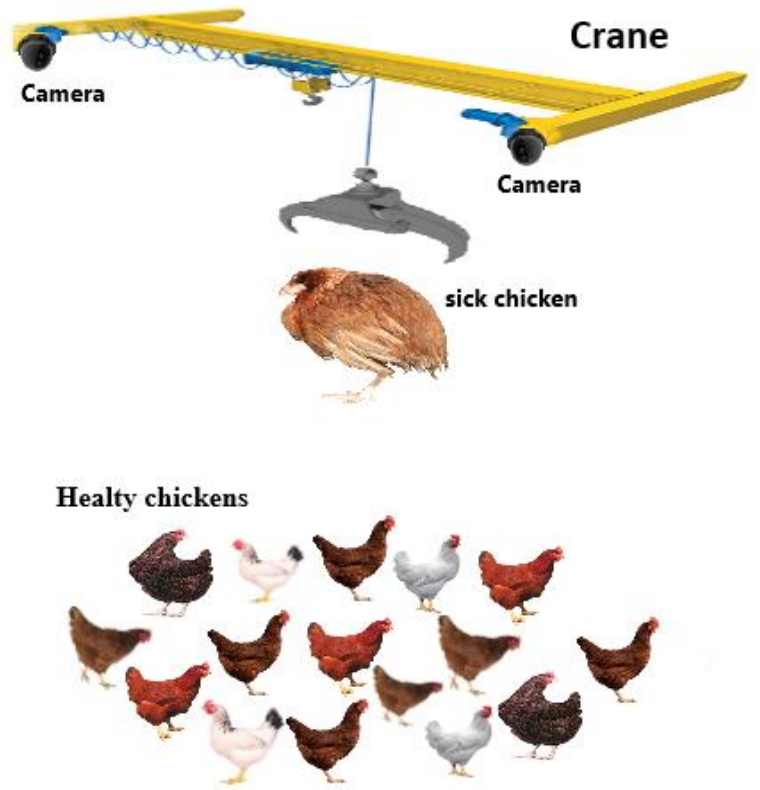

Figure 8. The system of automatically detect sick broilers

\section{RESULT and CONCLUSION}

The poultry diseases such as avian influenza that are a feature of fast-spread in farms seriously threatens both the economy and human health. Avian influenza must be detected early because it spreads rapidly. Earlier detection of poultry diseases has become more possible with the development of systems combining IPTs and AITs. In this paper, application of IPT based NNLVQ is successfully carried out for classification of broilers in terms of avian influenza. The model is conducted through 300 data of which visual features of broilers acquired using IPT. The training, testing and validating of the NNLVQ model is accomplished by using data of 260,20 and 20 visual data, respectively. In the training process, the NNLVQ model successfully classifies the broilers as healthy and sick with an accuracy of $99.616 \%$. The NNLVQ model classifies the outputs with accuracy of $100 \%$, for validating process. The proposed NNLVQ model can be integrated to a hardware system so as to automatically classify sick broilers in a farm. In addition, automatic classification of broilers in terms of different health problems can be made after NNLVQ are updated.

\section{REFERENCES}

[1] A. C. Fanatico, P. B. Pillai, J. L. Emmert, and C. M. Owens, "Meat quality of slow- and fast-growing chicken genotypes fed low-nutrient or standard diets and raised indoors or with outdoor access," Poult. Sci., vol. 86 , no. 10 , pp. 2245-2255, 2007, doi: $10.1093 / \mathrm{ps} / 86.10 .2245$.
[2] X. Zhuang, M. Bi, J. Guo, S. Wu, and T. Zhang, "Development of an early warning algorithm to detect sick broilers," Comput. Electron. Agric., 2018, doi: 10.1016/j.compag.2017.11.032.

[3] M. B. R. Mollah, M. A. Hasan, M. A. Salam, and M. A. Ali, "Digital image analysis to estimate the live weight of broiler," Comput. Electron. Agric., 2010, doi: 10.1016/j.compag.2010.02.002.

[4] H. R. Hemati Matin, A. A. Saki, M. Bayat Varkeshi, and H. Zare Abyaneh, "Comparison and validation of artificial intelligent techniques to estimate intestinal broiler microflora," Neural Comput. Appl., 2013, doi: 10.1007/s00521-012-1059-2.

[5] A. Aydin, "Development of an early detection system for lameness of broilers using computer vision," Comput. Electron. Agric., 2017, doi: 10.1016/j.compag.2017.02.019.

[6] D. F. Pereira, B. C. B. Miyamoto, G. D. N. Maia, G. Tatiana Sales, M. M. Magalhães, and R. S. Gates, "Machine vision to identify broiler breeder behavior," Comput. Electron. Agric., 2013, doi: 10.1016/j.compag.2013.09.012.

[7] P. F. P. Ferraz et al., "Predicting chick body mass by artificial intelligence-based models," Pesqui. Agropecu. Bras., 2014, doi: 10.1590/S0100-204X2014000700009.

[8] A. K. Mortensen, P. Lisouski, and P. Ahrendt, "Weight prediction of broiler chickens using 3D computer vision," Comput. Electron. Agric., 2016, doi: 10.1016/j.compag.2016.03.011.

[9] T. Zhang, M. Bi, J. Guo, and X. Zhuang, "Data for: Broiler chickens posture feature extraction and disease early-warning algorithm," $\begin{array}{lll}\text { Mendeley Data, V1, } & 2017 .\end{array}$ https://data.mendeley.com/datasets/txjj8mwtz6/1.

[10] T. Temel, "A new classification algorithm: Optimally Generalized Learning Vector Quantization (OGLVQ)," Neural Netw. World, 2017, doi: 10.14311/NNW.2017.27.031.

[11] S. Haykin, M. C. Publishing, J. Kacprzyk, and J. Wiley, "Neural Networks: A Comprehensive Foundation, Possibility Theory . An Approach to the Computerized Processing of Uncertainty ( Plenum the Management of Uncertainty, edited by L . A .," 1994.

[12] Ö. Dursun, S. Toraman, and A. Türkoğlu, "Comparison Of The Classification Performances Of Criminal Tendencies Of Schizophrenic Patients By Artificial Neural Networks And Support Vector Machine," Eur. J. Tech., vol. 7, no. 2, pp. 177-185, 2017.

[13] B. Kilic, "Impedance Image Reconstruction with Artificial Neural Network in Electrical Impedance Tomography," Eur. J. Tech., Dec. 2019, doi: 10.36222/ejt.650616.

\section{BIOGRAPHIES}

Ahmet Kayabasi received his B.S. and M.S. degrees in EEE from Selcuk University, Turkey, in 2001, 2005 respectively. In 2015, he received his Ph.D. degree in electrical and electronics engineering from Mersin University, Turkey. From 2001 to 2015, he was a lecturer in the Electronics and Automation Department of Selcuk University. He has been working as Associate Professor in the Department of Electrical and Electronics Engineering at Karamanoglu Mehmetbey University. His current research interests include image processing techniques, artificial intelligent, antennas, microstrip antennas, computational electromagnetic. 